\title{
Equação de difusão em coordenadas cilíndricas aplicada na secagem de sementes da Araucária (Araucaria angustifolia)
}

\author{
Célia M. R. Franco ${ }^{1}$ \\ UAFM/UFCG, Cuité, PB \\ Aluizio F. S. Júnior ${ }^{2}$ \\ UAFM/UFCG, Cuité, PB \\ Ivo D. Araújo ${ }^{3}$ \\ UAFM/UFCG, Cuité, PB
}

\begin{abstract}
Resumo. Neste trabalho estimou-se a variação da umidade no interior de sementes de uma árvore bem brasileira, Araucária (Araucaria angustifolia), durante o processo de secagem em camada fina. A Araucária corre risco de extinção e suas sementes (pinhões) recalcitrantes perdem o poder germinativo após processo de secagem, dificultando seu armazenamento e conservação da espécie. Com o objetivo de simular a distribuição de umidade no interior das sementes da Araucária foram propostos dois modelos matemáticos formulados a partir da equação de difusão bidimensional em coordenadas cilíndricas. O primeiro modelo envolve propriedades termofísicas constantes e a solução analítica foi obtida pelo método de separação de variáveis. O segundo modelo considera difusividade variável e a solução numérica foi obtida utilizando-se o método de volumes finitos, com uma formulação totalmente implícita. Os modelos pressupõem condição de contorno de terceira espécie e as sementes da Araucária foram representadas por um cilindro. A fim de validar os modelos, foram obtidos dados experimentais do teor de umidade de sementes da Araucária durante o processo de secagem contínua na temperatura de $70^{\circ} \mathrm{C}$. Para avaliar o efeito das considerações adotadas quanto à difusividade ser constante ou variável, um código computacional em linguagem Fortran foi utilizado para realização das otimizações e simulações. De acordo com os resultados, os modelos descrevem bem a cinética de secagem contínua de sementes da Araucária e podem ajudar a conhecer os efeitos da secagem sobre as propriedades químicas e biológicas do produto, uma vez que afetam os fenômenos de transferência de calor e massa. Foi observado que o modelo com a adoção do coeficiente de difusão dependente do teor de umidade local descreve melhor a cinética de secagem contínua de sementes da Araucária na temperatura de $70^{\circ} \mathrm{C}$.
\end{abstract}

Palavras-chave. Difusão de Massa, Modelagem Matemática, Difusividade Variável, Pinhão.

\section{Introdução}

Pinhão é a semente proveniente da Araucária (Araucaria angustifolia), encontrado na região Sul e em algumas partes do Sudeste do Brasil. Em São Paulo, é comum nas cidades serranas como Campos do Jordão. A Araucária, também conhecida como pinheiro-brasileiro ou pinheirodo-paraná, está em risco crítico de extinção, segundo a lista vermelha da União Internacional para a Conservação da Natureza e dos Recursos Naturais. Estima-se que atualmente a floresta de Araucárias ocupe apenas 3\% de sua área original. Um estudo publicado em 2019 prevê o fim da

\footnotetext{
${ }^{1}$ celia.maria@professor.ufcg.edu.br

2 aluizio.freire@professor.ufcg.edu.br

3ivo_dantas@outlook.com
} 
espécie até 2070 se nenhuma estratégia de conservação for posta em prática. A semente, o pinhão, possui alto valor nutricional e é utilizada na culinária regional e como complemento de renda de produtores locais.

Para garantir a qualidade da semente até a mesa do consumidor, é necessário estabelecer uma forma de conservação. Desde a antiguidade, a desidratação era usada na conservação de alimentos bem como a secagem de cereais pela ação do sol e do vento. Reduzir o teor de umidade das sementes diminui o risco de ataque de insetos e fungos, porém, o efeito do processo de secagem é alterado de acordo com as propriedades físico-química dos alimentos, interferindo assim na cinética de secagem [2].

O processo de secagem envolve transferência de calor e massa (umidade) entre um produto higroscópico e o ar de secagem. A análise do processo de secagem passa pela realização de experimentos e pelo desenvolvimento de modelos matemáticos capazes de predizer o processo. Os modelos matemáticos descrevem a perda de umidade ao longo do tempo, até a umidade de equilíbrio, avaliando assim a influência do processo de secagem [1]. Em geral, as pesquisas reportadas na literatura utilizam modelos empíricos para descrever a cinética de secagem da semente da Araucária [2,9]. Entretanto, modelos empíricos não fornecem indicações sobre os fenômenos de transporte de energia e de água no interior das sementes.

Neste sentido, existem várias teorias de secagem propostas na literatura que visam descrever o transporte de massa e calor em meios capilares porosos, como por exemplo a teoria de difusão líquida. Os modelos difusivos geralmente baseiam-se na difusão de líquido e/ou vapor no interior do produto, dependendo do tipo do material e do teor de umidade. Estes modelos descrevem as taxas de transferência de calor e massa como função da posição dentro do sólido e do tempo de secagem, e consideram as resistências aos fluxos de calor e massa externa e interna. Em geral, o coeficiente de difusão é considerado constante ou dependente da temperatura e/ou do teor de umidade do sólido. Para geometrias mais simplificadas, o problema pode ser resolvido analiticamente, o que normalmente pressupõe propriedades termofísicas constantes. Por outro lado, se o problema envolve propriedades termofísicas variáveis com condições de contorno gerais definidas em geometrias complexas, os métodos numéricos (elementos finitos, diferenças finitas e volumes finitos) são apropriados para obter a solução da equação de difusão. Soluções analíticas e/ou numéricas da equação de difusão, considerando o coeficiente de difusão constante ou variável, para diferentes geometrias (paralelepípedo, cilindro, esfera) e condições de contorno podem ser encontradas na literatura $[3,4]$.

Considerando a escassez de pesquisas relacionadas à modelagem matemática da secagem de sementes da Araucária, esse estudo objetiva-se propor dois modelos difusivos para descrever secagem contínua de sementes da Araucária na temperatura de $70^{\circ} \mathrm{C}$ e analisar a influência de propriedades termofísicas constantes ou variáveis na descrição do processo.

\section{Modelagem Matemática}

Para descrever o transporte de massa (umidade) durante o processo de secagem de sementes da Araucária (pinhões) considerou-se os fluxos nas direções radial e axial. A semente da Araucária foi representada por um cilindro de comprimento $L$ e raio $R$, com teor de umidade inicial $\Phi_{i}$ e supondo que existe simetria em relação ao eixo central e simetria radial em relação ao centro (Figura 1a). A equação de difusão bidimensional em coordenadas cilíndricas é dada da seguinte forma:

$$
\frac{\partial(\lambda \Phi)}{\partial t}=\frac{1}{r} \frac{\partial}{\partial r}\left(r \Gamma^{\Phi} \frac{\partial \Phi}{\partial r}\right)+\frac{\partial}{\partial y}\left(\Gamma^{\Phi} \frac{\partial \Phi}{\partial y}\right)
$$

onde $\Phi$ denota o teor de umidade no interior do produto, $\lambda$ e $\Gamma^{\Phi}$ são os coeficientes de transporte, $t>0$ é o tempo e $0<r<R$ e $-L / 2<y<L / 2$ são as coordenadas radial e axial, respectivamente. 


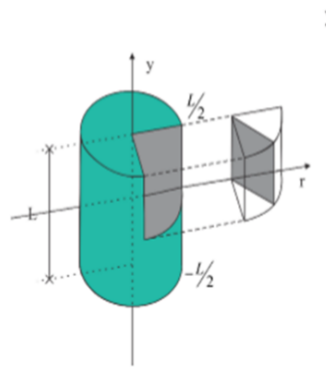

(a)

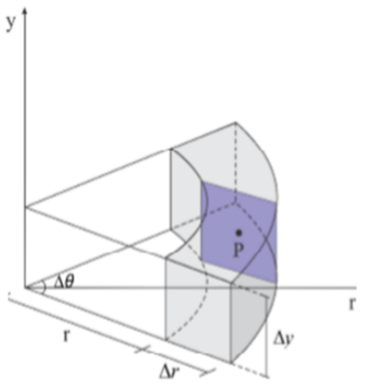

(b)

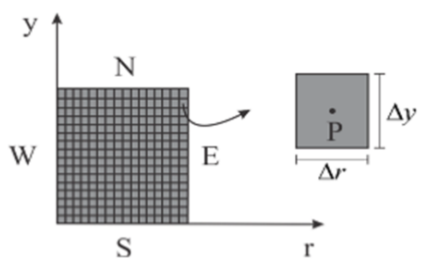

(c)

Figura 1: (a) Cilindro finito de comprimento $L$ e raio $R$. (b) Volume de controle para geometria cilíndrica. (c) Malha bidimensional para o caso do cilindro finito. Fonte: [6]

A condição de contorno de terceira espécie, condição inicial e simetria são estabelecidos como segue:

$$
\begin{gathered}
\Phi(r, y, 0)=\Phi_{i} \\
-\Gamma^{\Phi} \frac{\partial \Phi(R, y, t)}{\partial r}+h\left[\Phi_{\infty}-\Phi(R, y, t)\right]=0 \\
\frac{\partial \Phi(0, y, t)}{\partial r}=0, \quad \Phi(0, y, t) \neq \infty \\
-\Gamma^{\Phi} \frac{\partial \Phi(r, L / 2, t)}{\partial y}+h\left[\Phi_{\infty}-\Phi(r, L / 2, t)\right]=0 \\
\frac{\partial \Phi(r, 0, t)}{\partial y}=0
\end{gathered}
$$

onde $h$ é o coeficiente de transferência de massa convectivo e $\Phi_{\infty}$ é o teor de umidade na superfície do sólido.

A solução analítica da equação (1), para condição de contorno de terceira espécie e considerando $\lambda=1$ e $\Gamma^{\Phi}=D$ (difusividade efetiva de massa), é dada por [3]:

$$
\frac{\bar{\Phi}-\Phi_{\infty}}{\Phi_{i}-\Phi_{\infty}}=\sum_{n=1}^{\infty} \sum_{m=1}^{\infty} B_{n, 1} B_{m, 2} \exp \left[-\left(\frac{\mu_{n, 1}^{2}}{R^{2}}+\frac{\mu_{m, 2}^{2}}{(L / 2)^{2}}\right) D t\right]
$$

onde

$$
\begin{gathered}
B_{n, 1}=\frac{4 B i_{1}^{2}}{\mu_{n, 1}^{2}\left(\mu_{n, 1}^{2}+B i_{1}^{2}\right)}, \quad B_{m, 2}=\frac{2 B i_{2}^{2}}{\mu_{m, 2}^{2}\left(B i_{2}^{2}+B i_{2}+\mu_{m, 2}^{2}\right)} \\
B i_{1}=\frac{h R}{D} \quad \text { e } \quad B i_{2}=\frac{h(L / 2)}{D}
\end{gathered}
$$


sendo $B i_{1}$ o número de Biot referente ao cilindro infinito e $B i_{2}$ o número de Biot referente à parede infinita, $\mu_{n, 1}$ e $\mu_{m, 2}$ são as raízes das equações características para o cilindro infinito e para a parede infinita, respectivamente.

O método utilizado para obter solução numérica da equação de difusão foi o método dos volumes finitos $[4,5]$ e as seguintes hipóteses foram admitidas: o cilindro é homogêneo e isotrópico; o único mecanismo de transporte de massa no interior do cilindro é a difusão; a difusividade efetiva pode variar durante a difusão, e o coeficiente de transferência convectiva de massa permanece constante.

A discretização inicia-se decompondo o domínio contínuo em um número finito de subdomínios $V_{i}$ com $i=1, \ldots, N$, chamados volumes de controle. A partir do cilindro é destacada uma repartição como mostra a Figura 1(a) e, devido as hipóteses de simetria, considerou-se apenas o retângulo, no qual foi criada uma malha uniforme, como é possível observar na Figura 1(c). O volume de controle definido para cada elemento da malha é ilustrado na Figura 1(b). Estes volumes de controle são caracterizados por um ponto onde as variáveis desconhecidas são calculadas, os quais são denotados pontos nodais. Na Figura 1(c) é apresentado um elemento de malha uniforme, onde é destacado o ponto nodal $P$, a largura $(\Delta r)$ e comprimento $(\Delta y)$. A malha bidimensional considerada possui 9 (nove) tipos de volumes de controle que são mostrados na Figura 2.

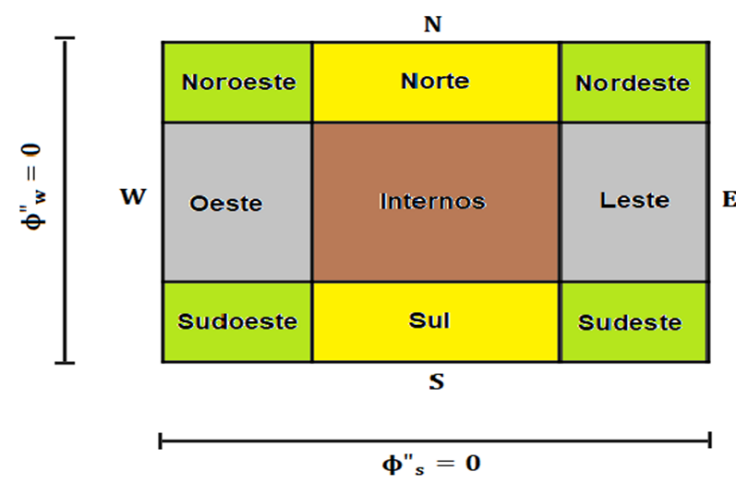

Figura 2: Tipos de volumes de controle da malha bidimensional.

Como o problema em estudo encontra-se em regime transiente, é necessário saber qual instante no intervalo de tempo será considerado para expressar as derivadas espaciais. Para isso, utiliza-se interpolação no tempo da grandeza de interesse $\Phi$.

Neste trabalho, considerou-se a formulação totalmente implícita na qual a variável é avaliada no final do intervalo de tempo. Uma das principais características desta formulação é a sua estabilidade incondicional, isto é, qualquer intervalo de tempo pode ser considerado sem interferir na convergência da solução.

Integrando a equação (1) no espaço $r_{p} \Delta r \Delta y$ e no tempo $\Delta t$ e usando as aproximações das derivadas, obtém-se:

$$
\left(\lambda_{P} \Phi_{P}-\lambda_{P}^{0} \Phi_{P}^{0}\right) \frac{r_{p} \Delta r \Delta y}{\Delta t}=\left(\left.r_{e} \Gamma_{e}^{\Phi} \frac{\partial \Phi}{\partial r}\right|_{e}-\left.r_{w} \Gamma_{w}^{\Phi} \frac{\partial \Phi}{\partial r}\right|_{w}\right) \Delta y+\left(\left.\Gamma_{n}^{\Phi} \frac{\partial \Phi}{\partial y}\right|_{n}-\left.\Gamma_{s}^{\Phi} \frac{\partial \Phi}{\partial y}\right|_{s}\right) r_{p} \Delta r
$$

onde o sobrescrito 0 indica o início do intervalo de tempo, os subscritos $e, w, n$, e $s$ são, respec- 
tivamente, o leste, oeste, interfaces norte e sul; enquanto $P$ denota o ponto nodal do volume de controle.

A partir da equação (10) são obtidas as equações discretizadas para todos os tipos de volumes de controle $[4,7]$.

\section{Resultados e Discussão}

Considerando o primeiro modelo, a solução analítica da equação de difusão para geometria cilindro finito e condição de contorno de terceira espécie foi ajustada aos dados experimentais de secagem de sementes da Araucária na temperatura de $70^{\circ} \mathrm{C}$. Na simulação foram utilizadas as dimensões da semente úmida in natura para obter as dimensões equivalentes do cilindro finito: $L=50,212 \mathrm{~mm}$ e $R=8,77 \mathrm{~mm}$. A grandeza de interesse é $\Phi=M$, onde $M$ denota o teor de umidade dos pinhões. O teor de umidade médio adimensional é denotado por $\bar{M}^{*}$.

A Figura 3a mostra a comparação entre os valores simulados e experimentais do teor de umidade médio adimensional dos pinhões com casca durante a secagem contínua em camada fina. Neste caso, os parâmetros termofísicos são considerados constantes. O coeficiente de difusão $(D)$, o coeficiente de transferência de massa convectivo $(h)$ e o número de Biot $(B i)$ foram obtidos e alguns parâmetros estatísticos que medem a qualidade do ajuste do modelo aos dados experimentais são apresentados na Tabela 1. Para tanto, utilizou-se o software Convective [8].

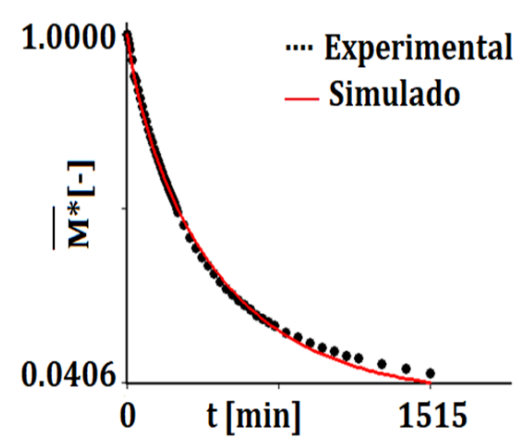

(a)

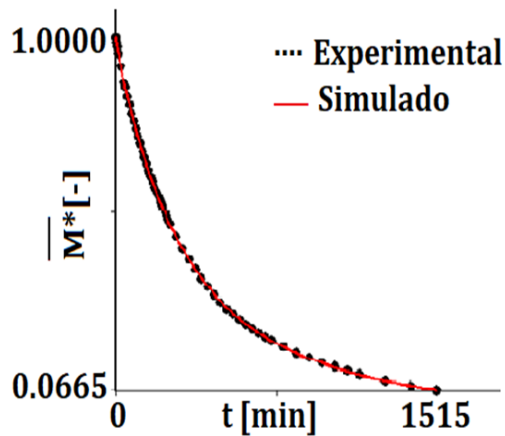

(b)

Figura 3: Comparação entre os teores de umidade médio adimensional teórico e experimental durante a secagem contínua de pinhões: (a) solução analítica (b) solução numérica.

Tabela 1: Resultados da otimização utilizando o software Convective.

\begin{tabular}{ccccc}
\hline$D\left(\mathrm{~m}^{2} / \mathrm{min}\right)$ & $h(\mathrm{~m} / \mathrm{min})$ & $B i$ & $\chi^{2}$ & $R^{2}$ \\
\hline $3,8302 \times 10^{-08}$ & $1,7906 \times 10^{-05}$ & 4,1 & $8,003244 \times 10^{-03}$ & 0,998258 \\
\hline
\end{tabular}

Observando o gráfico gerado pela simulação no Convective (Figura 3a) e a Tabela 1, é possível perceber que o modelo com difusividade constante se ajustou bem aos dados experimentais de secagem contínua de sementes da Araucária. No entanto, uma inspeção da Figura 3(a) mostra que, 
na parte final do processo de secagem, a difusividade de massa deve ser menor que o valor obtido com o modelo que considera propriedades termofísicas constantes. Isso significa que, quando o teor de umidade diminui, a difusividade de massa também deve diminuir. Assim, se a difusividade de massa no interior do produto varia pouco, para todo instante $t$, a solução do modelo com propriedades constantes funciona bem. Por outro lado, em algumas situações físicas a difusividade de massa constante é inadequado para descrever a cinética de secagem, como visto na Figura 3 a.

Para estudar o efeito da difusividade $(D)$ variável com o teor de umidade, utilizou-se a solução numérica da equação de difusão (segundo modelo), considerando condição de contorno de terceira espécie. Uma vez estabelecida a malha (15 x 10) e o número de passos de tempo (1500), procurou-se determinar uma expressão adequada para descrever a variação da difusividade efetiva de massa em função do teor de umidade durante a secagem contínua de sementes da Araucária, além de obter um valor ótimo para o coeficiente de transferência de massa convectivo $(h)$. Para isto, utilizou-se o software Cilindro Finito descrito em [7], que disponibiliza uma biblioteca com 30 (trinta) expressões para a difusividade. Neste trabalho, foram testadas 5 (cinco) funções que estão na biblioteca do software e são apresentadas na Tabela 2.

Tabela 2: Expressões para difusividade.

\begin{tabular}{cc}
\hline Notação & Função \\
\hline$F_{1}$ & $b$ \\
$F_{2}$ & $a x+b$ \\
$F_{3}$ & $a x^{2}+b$ \\
$F_{4}$ & $b \exp (a x)$ \\
$F_{5}$ & $b \cosh (a x)$ \\
\hline
\end{tabular}

Os resultados dos valores ótimos dos parâmetros $a$ e $b$ foram obtidos por otimização e estão apresentados na Tabela 3 para as cinco funções, juntamente com o coeficiente de transferência de massa convectivo $(h)$ e os parâmetros estatísticos: coeficiente de determinação $\left(R^{2}\right)$ e qui-quadrado $\left(\chi^{2}\right)$. Os resultados encontram-se em ordem de melhor ajuste aos dados experimentais. Analisando os resultados apresentados na Tabela 3, foi possível obter valores do qui-quadrado menores quando comparado com o qui-quadrado obtido com a utilização da solução analítica (primeiro modelo), o que indica que a solução numérica, que considera difusividade variável, é mais adequada para descrever a cinética de secagem. Com relação as funções testadas, todas apresentaram coeficiente de determinação $\left(R^{2}\right)$ próximo de 1 e o valor do qui-quadrado $\left(\chi^{2}\right)$ relativamente baixo, sendo a função $F_{2}$ a que apresenta os melhores indicadores estatísticos. A Figura 3 b mostra a comparação entre os dados experimentais e os dados obtidos na simulação considerando a difusividade variável dada pela função $F_{2}$, para secagem contínua de sementes da Araucária. Neste caso, a difusividade de massa efetiva pode ser considerada constante ou variável (em função do teor de umidade local), expandindo o limite de validade da solução obtida.

Tabela 3: Resultados da otimização utilizando solução numérica.

\begin{tabular}{|c|c|c|c|c|c|}
\hline Função & $a$ & $b$ & $h(\mathrm{~m} / \mathrm{min})$ & $\chi^{2}$ & $R^{2}$ \\
\hline$F_{2}$ & $1,2646 \times 10^{-07}$ & $1,0463 \times 10^{-08}$ & $1,3267 \times 10^{-05}$ & $4,3017 \times 10^{-04}$ & 0,99989 \\
\hline$F_{3}$ & $2,9132 \times 10^{-07}$ & $2,3038 \times 10^{-08}$ & $1,2239 \times 10^{-05}$ & $5,8600 \times 10^{-04}$ & 0,99986 \\
\hline$F_{4}$ & 2,8522 & $2,0559 \times 10^{-08}$ & $1,2375 \times 10^{-05}$ & $6,6035 \times 10^{-04}$ & 0,99984 \\
\hline$F_{5}$ & 0,1003003 & $3,5322 \times 10^{-08}$ & $1,9664 \times 10^{-05}$ & $7,2468 \times 10^{-03}$ & 0,99831 \\
\hline$F_{1}$ & 0 & $3,6190 \times 10^{-08}$ & $1,9238 \times 10^{-05}$ & $7,2600 \times 10^{-03}$ & 0,99831 \\
\hline
\end{tabular}




\section{Conclusões}

Foi possível descrever o teor de umidade de sementes da Araucária, durante processo de secagem na temperatura de $70^{\circ} \mathrm{C}$, utilizando dois modelos difusivos. Considerou-se soluções analítica e numérica da equação de difusão e, a partir das simulações realizadas, obteve-se o coeficiente de transferência de massa convectivo e foi determinada uma expressão para descrever a difusividade de massa em função do teor de umidade local. Os indicadores estatísticos obtidos com o ajuste da solução numérica apresentaram valores significativamente melhores quando comparados aos obtidos com o ajuste realizado no software convective, que considera difusividade constante. Portanto, destaca-se a importância do estudo de métodos numéricos na implementação de modelos matemáticos que consideram propriedades termofísicas variáveis para descrever processos de secagem com mais eficiência e aproximação da situação real. Os modelos matemáticos propostos nesse trabalho podem ser utilizados para simular a distribuição de umidade no interior de sementes da Araucária em qualquer instante, fornecendo informações sobre o que realmente acontece com o produto durante processos de secagem.

\section{Referências}

[1] Franco, C. M. R. e Lima, A. G. B. Modelagem matemática e simulação computacional da secagem contínua e intermitente de sólidos elipsoidais, Proceeding Series of the Brazilian Society of Computational and Applied Mathematics, volume 6, 2018. DOI: 10.5540/03.2018.006.02.0298.

[2] Martim, C. C., Silva, S. G., Ferneda, B. G., Luz, E. G. e Justi, J. Curva de secagem e contração volumétrica para o pinhão da Araucária (Araucária angustifólia). Enciclopédia Biosfera, 2019. DOI: 10.18677/EnciBio_2019B18.

[3] Luikov, A. V. Analytical heat diffusion theory. Academic Press, New York and London, 1968.

[4] Maliska, C. R. Transferência de calor e mecânica dos fluidos computacional. LTC Editora S.A., Rio de Janeiro, 2004.

[5] Patankar, S. V. Numerical heat transfer and fluid flow. Hemisphere Publishing Corporation, New York, 1980.

[6] Silva Junior, A. F., Silva, W. P., Aires, J. E. F., Aires, K. L. C. A. Farias and Castro, D. S. Osmotic dehydration kinetics of banana slices considering variable diffusivities and shrinkage International Journal of Food Properties, volume 20, 2017. DOI: 10.1080/10942912.2016.1209215.

[7] Silva Junior, A. F., Silva, W. P. Desidratação osmótica e secagem complementar de fatias de banana: desenvolvimento de ferramentas numéricas e computacionais. Novas Edições Acadêmicas, Beau Bassin, 2018.

[8] Silva, W. P., Silva, C. M. D. P. S.Convectivo - Adsorção e Dessorção, Versão 2.2. http://zeus.df.ufcg.edu.br/labfit/Convective.htm, 2009.

[9] Vieira, M. E., Kitawara, A. M., Fernandes, B. N. e Okabe, J. N. Estudo da cinética de secagem de pinhão. CONIC SEMESP, 17 Congresso Nacional de Iniciação Científica, 2017, ISSN: $2357-8904$. 